\title{
Prairie naturelle, « Campero » et ingénieur
}

Deux conceptions de l'élevage en Uruguay

Native grassland, the "campero " and the engineer. Two approaches to animal farming in Uruguay

\section{Maria Fernanda de Torres Alvarez}

\section{(2) OpenEdition \\ Journals}

Édition électronique

URL : https://journals.openedition.org/tc/7402

DOI : $10.4000 /$ tc. 7402

ISBN : 0248-6016

ISSN : 1952-420X

Éditeur

Éditions de l'EHESS

\section{Édition imprimée}

Date de publication : 1 mai 2015

Pagination : 74-91

ISBN : 9782713224874

ISSN : 0248-6016

Référence électronique

Maria Fernanda de Torres Alvarez, «Prairie naturelle, «Campero » et ingénieur », Techniques \& Culture [En ligne], 63 | 2015, mis en ligne le 15 décembre 2018, consulté le 29 septembre 2022. URL : http:// journals.openedition.org/tc/7402; DOI : https://doi.org/10.4000/tc.7402 


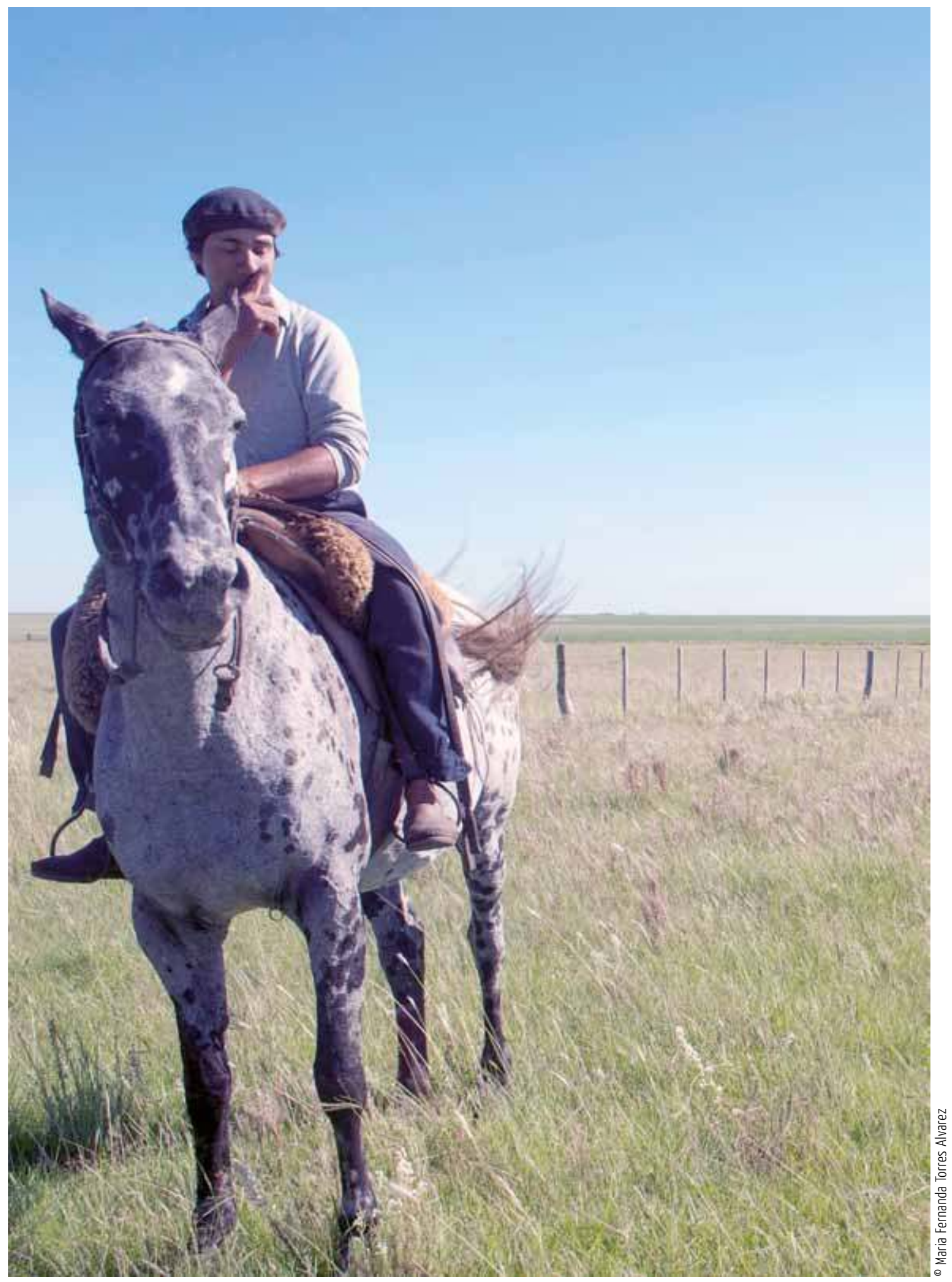




\section{PRAIRIE NATURELLE, « CAMPERO » ET INGÉNIEUR}

\section{Deux conceptions de l'élevage en Uruguay}

Les pâturages naturels du Rio de la Plata occupent 70 millions d'hectares entre la pampa Argentine, le Rio Grande do Sul au Brésil et l'Uruguay, ces derniers couvrant 11 millions d'hectares. C'est la région de pâturages naturels la plus étendue du monde, mais elle est de plus en plus menacée par l'expansion de l'irrigation, de la plantation forestière et de l'agriculture (Paruelo \& al. 2006; Jobbágy \& al., 2006). En Uruguay, la préservation de cet écosystème ne résulte pas de politiques publiques environnementales innovantes, mais bien plutôt d'une longue activité d'élevage qui a modelé le territoire uruguayen de prairies naturelles. Cet écosystème caractérise le paysage national depuis la colonisation européenne, au xv ${ }^{e}$ siècle. Durant le xxle siècle, l'élevage sur pâturage naturel a occupé jusqu'à $90 \%$ du territoire national avant que l'essor de l'agriculture et de la sylviculture, favorisés par les politiques d'investissements étrangers, ne le limite à $60 \%$.

La modernisation de l'élevage a placé au cour des débats la question de l'alimentation animale. À ce moment-là, est apparu un « problème fourrager » qui renvoie à la nécessité d’augmenter la productivité de la prairie naturelle associée à l'élevage traditionnel. Le savoir agronomique fait valoir un point de vue qui s'oppose radicalement à celui des travailleurs agricoles en charge de l'élevage, et notamment ceux qui assurent la « recorrida», cette tâche quotidienne, peu visible et peu valorisée, dédiée à la surveillance des parcelles dans lesquelles vivent les vaches. Deux points de vue s'opposent ainsi nettement. Ils mettent chacun en tension des conceptions très différentes du travail d'élevage, de ses exigences et supposent des rapports aux bêtes et aux milieux naturels, en l'occurrence la pampa, également contrastés. C'est à l'analyse de ces deux points de vue sur le pâturage et sur l'élevage que nous procéderons ici, en présentant notamment les critiques que ces deux

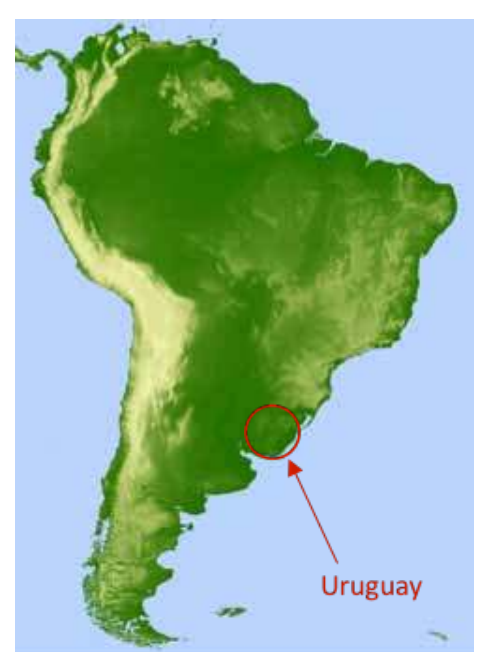




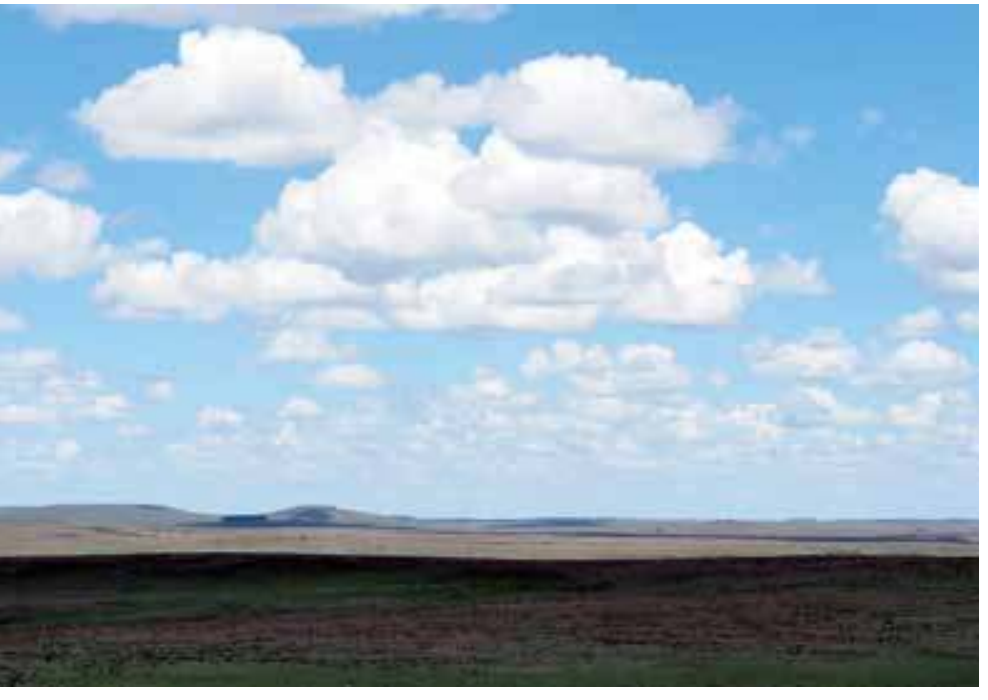

Prairie naturelle lisse

L'élevage à ciel ouvert s'étend sur des espaces lisses, où la terre s'ouvre vers le ciel.

\section{Prairie naturelle} Dans ces campos, le relief est plus énergique, de petites forêts se produisent plus fréquemment et dominent le paysage des prairies naturelles.

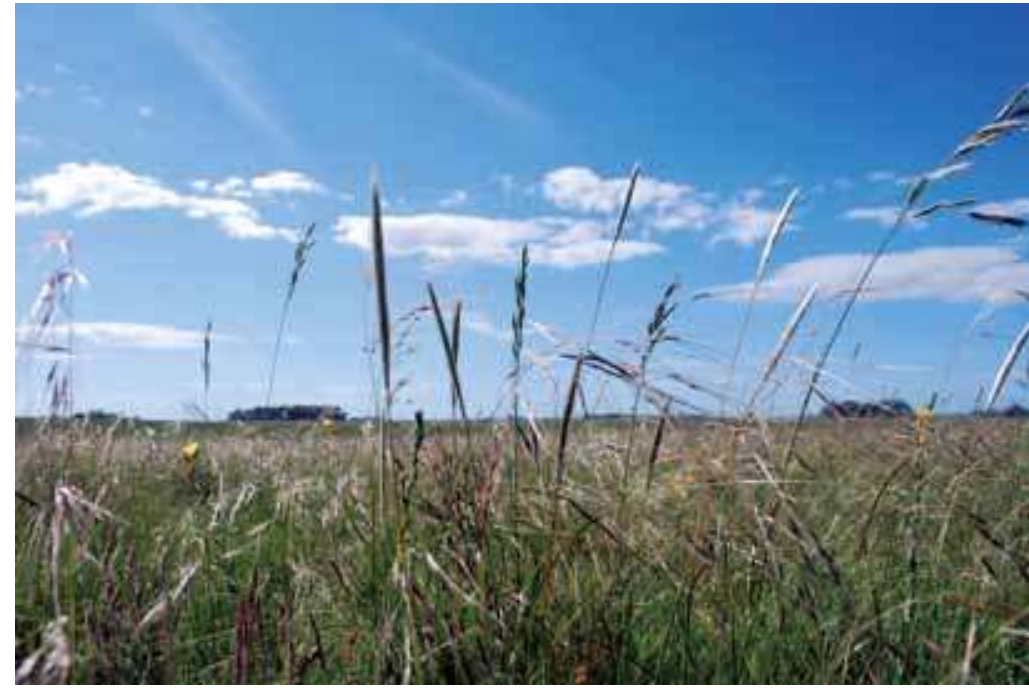

Prairie naturelle dans le basalte

Novembre 2012 dans la prairie naturelle, Département de Salto, Route 31, Uruguay.

Un écosystème unique au monde

Septembre 2012 dans Departamento de Rivera, Route 31.

«Cet écosystème est tout aussi précieux pour la planète que la jungle et en plus il produit de la viande ! Je ne comprends pas pourquoi personne ne dit pas qu'il faut sarrêter de labourer, de tout remuer, d'épandre des herbicides... II s'agit d'un écosystème unique au monde", s'indigne un ouvrier agricole de 45 ans, dont 22 d'expérience professionnelle.

Prairie naturelle

Novembre 2012 sur la Route 31 Département de Salto.

L'élevage à ciel ouvert, dans du sol de basalte.

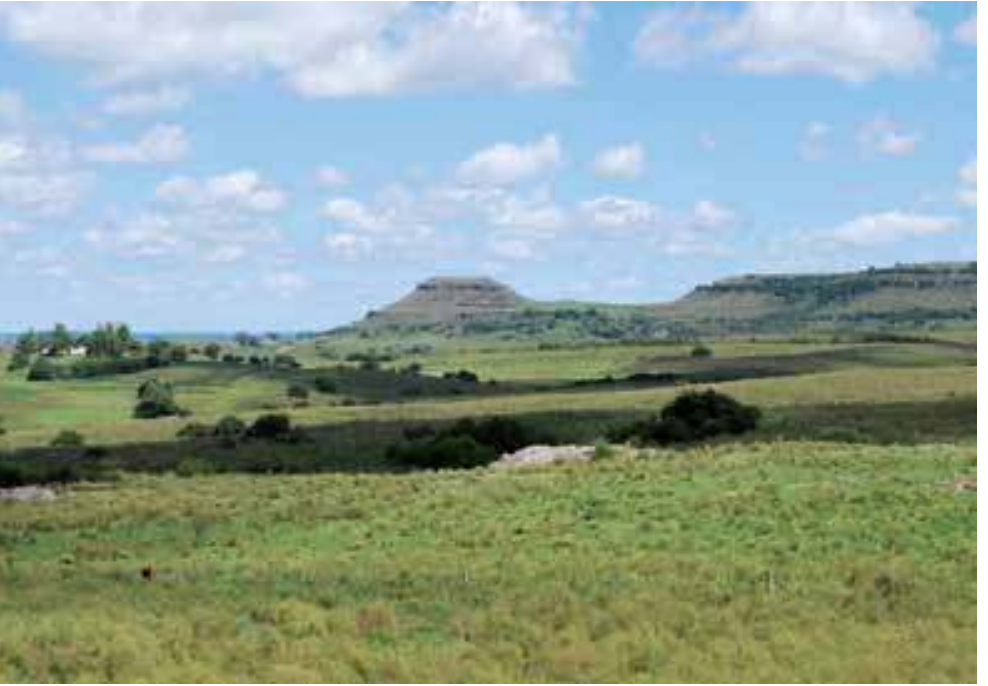


mondes s'adressent réciproquement. Nous mobiliserons un cadre analytique combinant anthropologie de la nature et phénoménologie telle qu'il est développé par Ingold (2010) et en faisant notre la distinction qu'il opère entre deux paradigmes caractérisant deux rapports au monde très contrastés que l'on retrouve dans les deux façons d'appréhender la prairie. La première (growing) caractérise un certain rapport au vivant en lien avec le fait d'élever, de faire croître les animaux, la seconde (making) renvoie à un processus de production évoquant le monde industriel et une vision moderniste de l'élevage.

La réflexion présentée dans cet article se fonde sur un travail ethnographique conduit dans le Nord de l'Uruguay, entre juin et novembre 2008 et 2009, dans une région caractérisée par les formes d'élevage les plus traditionnelles et moins intensives du pays. Une série d'entretiens conduits lors de séjours d'une semaine dans chacune des 35 exploitations agricoles enquêtées a permis de décrire les activités et l'organisation de l'élevage. Ce volet ethnographique est complété par l'analyse d'archives gouvernementales datant de la première moitié $\mathrm{du} \mathrm{xx}$ e siècle et qui permettent de retracer l'histoire de l'innovation technique dans les pâturages naturels. Enfin, la réflexion s'ancre sur l'exploitation d'un corpus de textes, les Contributions, qui constitue un ensemble de propositions issues de la recherche agricole et que l'on peut considérer comme la première recherche scientifique, portant sur l'écologie et la botanique de la flore indigène de l'Uruguay.

Après avoir présenté à grands traits les caractéristiques de l'élevage traditionnel, nous reviendrons sur la vision modernisatrice portée par les agronomes en insistant sur une technique d'amélioration du pâturage, le roulage, très fortement controversée par les éleveurs sur prairies naturelles. Puis, après avoir ensuite montré en quoi les « Contributions » ont constitué un apport majeur à la description, la reconnaissance et la défense de l'élevage traditionnel, nous pourrons alors revenir sur ce qui caractérise une tache très particulière de l'élevage traditionnel: la « recorrida ». Nous soulignerons les compétences qu'elle requière et qui fondent un rapport très singulier entre hommes, bêtes et milieux naturels, tout à l'opposé du paradigme modernisateur et techniciste des agronomes.

\section{L'élevage sur prairie naturelle: un travail (rendu) invisible dans la pampa}

La surface des exploitations agricoles varie entre 100 et 500 hectares pour les petites exploitations, de 500 à 2000 hectares pour les moyennes et plus de 2000 ha pour les grandes propriétés. Le territoire de l'élevage, la pampa, se caractérise par une faible densité de population rurale ( 0.025 habitants/hectare), associée à des systèmes d'exploitation agricoles (estancias) de diverses tailles, des villages et des villes moyennes, l'ensemble permettant une reproduction sociale et biologique du système écologique et social de l'élevage. On compte 38000 exploitations d'élevage, 8,3 millions de bovins et 9,3 millions d'ovins. L'élevage avec agriculture est minoritaire : il ne concerne que 8000 exploitations pour 2 millions de bovins et 1,5 million d'ovins. Les exploitations bovines concernent les exploitations de 1000 ha ou plus.

Lélevage extensif est une activité très saisonnière, caractérisée par un travail cyclique de faible intensité où la plupart des activités se concentrent pendant la période de fécondation 


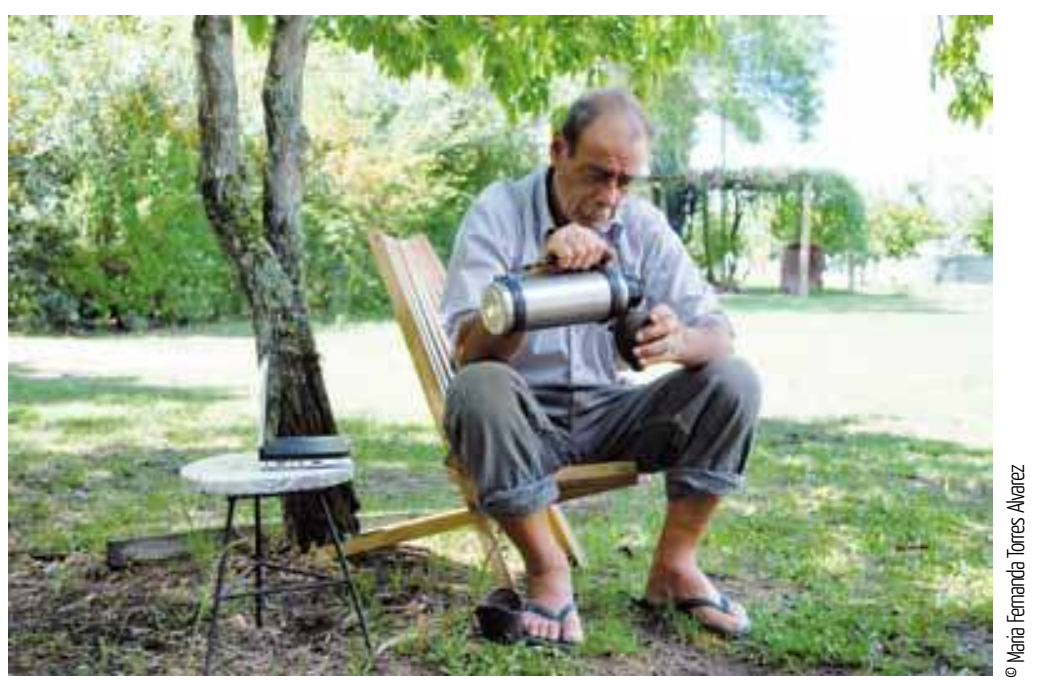

Peón après le Recorrida

Septembre 2012, Département Rivera (Route 27), l'Uruguay. Après la recorrida, le péon retire ses bottes et prépare le mate, l'infusion traditionnelle. L'heure est au repos à l'ombre d'un arbre. (artificielle et naturelle) des femelles (avril), la naissance des veaux (août) et le sevrage (mai). Après le sevrage, commence une période de trois ans, ou un peu moins, au terme de laquelle l'animal peut soit aller directement à l'abattoir s'il a atteint le poids requis $(450 \mathrm{~kg})$, soit passer par la phase terminale d'engraissement. Les trois premières étapes de ce cycle d'élevage nécessitent des compétences et des qualifications techniques reconnues d'un point de vue agronomique académique, au contraire de l'étape de croissance de l'animal. En effet, la sociologie rurale et la sociologie du travail (Piñeiro, 2008; Moreira, 2010) ont renforcé l'idée déjà fortement présente en agronomie, d'un travail non productif, arguant du fait que c'est la nature seule qui fait tout le travail.

Le travail principal de la phase de croissance est appelé Recorrida, ce qui signifie " parcours de surveillance ». Il s'agit d'une tâche d'astreinte, effectuée tous les jours ou deux fois par jour, à cheval, sur tout ou partie de l'exploitation. Ce travail est réalisé par le patron s'il habite l'estancia ou par les travailleurs permanents ou saisonniers (campero, péon). Il consiste principalement à collecter une série d'informations concernant le campo natural et les animaux. L'attention se focalise sur le bien-être des bêtes, l'état des clôtures, du pâturage naturel et des sols, ainsi que sur la disponibilité en eau. La veille météorologique, c'est-à-dire, l'observation du ciel qui implique le développement d'une compétence corporelle totale. Le péon ou le campero communiquent les données qu'ils recueillent lors de la recorrida au propriétaire ou au gérant, lesquels prendront les décisions qui s'imposent: fixer une clôture, guérir un animal malade, chercher des bêtes perdues chez les voisins, effectuer des rotations de parcelles lorsque l'herbe manque, mettre un pâturage en jachère, etc.

Les métiers de l'élevage sont très hiérarchisés: le péon est la base de la pyramide, suivi par le contremaître (capataz) et le gestionnaire (l'administrateur ou le patron) de l'exploitation agricole. La recorrida est faite habituellement par le péon, parfois avec le contremaître et dans le cas de l'élevage familial, par le peón et le patron (Moreira, 2010). Le peón n'a pas de formation scolaire formelle (niveau d'étude : école primaire) et ses compétences sont acquises empiriquement par l'exécution des tâches. Au bas de la chaîne hiérarchique, il est le moins bien payé, même depuis l'amélioration des conditions de travail et l'approbation des huit heures journalières, votées par l'actuel gouvernement. Travailleur socialement dévalorisé, mal, voire pas reconnu professionnellement, le péon effectue un travail décrit comme ne nécessitant pas de qualifications ni de compétences particulières, puisque c'est « la nature qui fait tout ». Le travail de la recorrida, comme le rappelle le sens du terme (« parcours de surveillance »), est avant tout un travail d'observation du bétail et des prairies. Il n'en mobilise pas moins un registre de compétences et de connaissances qui repose sur une vision de l'élevage qui nous le verrons dans le dernier temps de cet article qui entre en tension avec le point de vue des agronomes planificateurs qu'il nous faut maintenant présenter. 


\section{Produire de la viande, améliorer les pâturages: un point de vue d'agronome}

Le secteur primaire agricole a toujours constitué une activité à part dans l'économie nationale, historiquement marquée par la mobilisation de connaissances scientifiques afin d'augmenter la production. Ce fut en particulier le cas de l'élevage, dont la production a été rapidement tournée vers l'exportation. Depuis le début du xx siècle, l'Uruguay s'est peu à peu convaincue qu'il était nécessaire d'améliorer les pâturages naturels qui poussaient spontanément sur ses sols, considérés comme dépourvus de valeur productive et botanique. En effet, la nouvelle ingénierie agronomique ${ }^{1}$ concentrait de façon majoritaire ses diagnostics sur le problème fourrager national (Böerger, 1939). En d'autres termes, le caractère homogène des espèces du tapis végétal, leur faible valeur nutritionnelle et la possibilité de l'améliorer grâce au labour pour la culture fourragère, constituent un véritable consensus technique national (de Torres, 2013). «Les pâturages qui existent en Uruguay ne sont pas assez bons pour l'Uruguay » voilà comment, en 1952, le technicien néo-zélandais engagé par la FAO-BIRD a commencé sa conférence, dédiée à la modernisation de l'élevage. Ces suggestions amplifiaient la thèse du problème fourrager qui allait dans le sens d'une substitution des espèces et l'épandage d'engrais, dessinant les futurs paysages agraires industriels (de Torres $\mathcal{E}$ al., 2013).

La base de ce consensus n'était pas tant fondée sur les données empiriques et les caractéristiques réelles de l'écosystème local, que sur la comparaison des rendements et de l'organisation de l'élevage-viande avec l'Allemagne, la Nouvelle-Zélande, l'Australie et les États-Unis. La modernisation de l'élevage nécessitait la mise en place de bovins de race et de pâturages optimisés pour répondre au rythme et à la qualité exigée par l'industrie de la viande. L'Uruguay avait déjà engagé un processus de métissage des races créoles avec des races pures britanniques (Hereford et Shorton). Celles-ci exigent un accès constant à des fourrages de qualité, pour assurer des performances zootechniques et conformations de carcasse en adéquation avec l'exigence de l'industrie. Lagronomie est apparue comme un moteur de la richesse nationale, par la transformation des sols et la maximisation de l'utilisation de la terre. En fait, le problème de la grande propriété avait été réglé non par la discussion de la propriété privée de la terre, mais par le fondement de l'utilité de cette propriété.

La conviction de la faible valeur intrinsèque et productive des pâturages naturels, sans aucune preuve empirique, permettra aux nouveaux agronomes de commencer un combat pour

\section{Journée technique avec les éleveurs}

Septembre 2012 dans le Plan Agropecuario et l'Association Rural deTacuarembó Route 26, I'Uruguay.

Les journées de vulgarisation permettent de mesurer le fossé qui sépare camperos et ingénieurs. La qualification des espèces végétales de la prairie naturelle est à ce titre éloquent. L'utilisation de différents noms (commerciaux, vernaculaires ou scientifiques) complique les échanges. Par exemple, pour les éleveurs et les camperos, la patte de poulet est l'un des pâturages à haut rendement, qui compte plusieurs espèces, que les agronomes identifient quant à eux, de leur nom savant (Paspalum dilatatum et Cynodon dactylon) au risque de ne pas être compris des éleveurs.

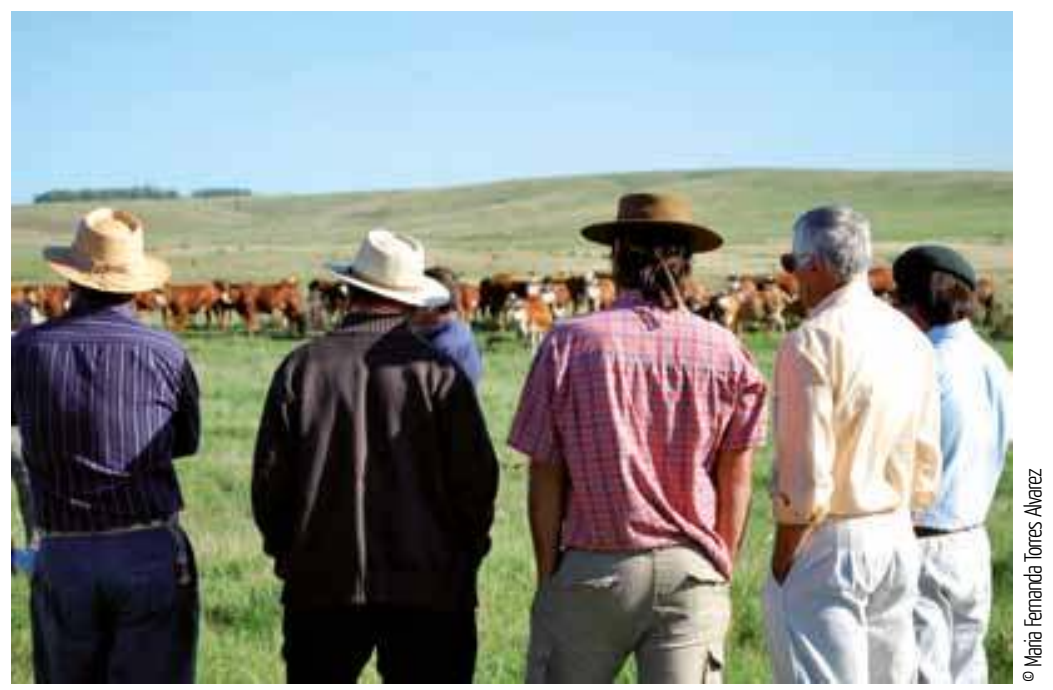


la transformation des zones d'élevage en zones agricoles, de maximiser la productivité grâce à l'introduction de technologies importées des centres de référence mondiaux. L'idée de la transformation - ici synonyme d'amélioration - est bien au cour du savoir de l'ingénieur et des dynamiques souhaitées pour l'élevage. Elle devient le mot d'ordre d'une profession, entièrement tendue vers une économie de marché très concurrentielle.

« l'ingénieur agronome se forme pour transformer, la formation d'un ingénieur agronomique consiste essentiellement à transformer, il s'agit de prendre une réalité et de la transformer... l'emblème a été de faire des pâturages, de faire des cultures, tous les changements technologiques devaient permettre d'augmenter les profits et, en plus, la stagnation était liée à un manque de ce type de changements (...), nous sommes sortis de la faculté comme des soldats de la transformation... $»^{2}$.

Les agronomes se sont alors intéressés au pâturage et ont recommandé que des prairies artificielles soient implantées conformément à la technique du roulage. Cette technique de travail du sol est destinée à préparer le semi du couvert végétal, en espèces productives, souvent exotiques. Au moyen d'un lourd rouleau de fonte, le couvert végétal est rompu, débarrassé de ses mottes, nivelé et légèrement tassé pour favoriser le contact des graines avec le sol et limiter l'évaporation. Le projet néo-zélandais introduit dans les années soixante (pâturages artificiels, ensemencement sur couverture et engrais phosphatés) a ainsi été adopté. Cette adoption fut moindre dans le Nord du pays, avec la seule incorporation d'aspects qui n'impliquaient pas de rupture définitive de la couverture du sol. Lors de l'année 2008-2009, la proportion de terres ainsi améliorées rapportées à la superficie totale des pâturages était à Tacuarembó de $8 \%$, de 10,6 \% à Rivera et de $5,4 \%$ pour Salto (MGAP, 20111: 74).

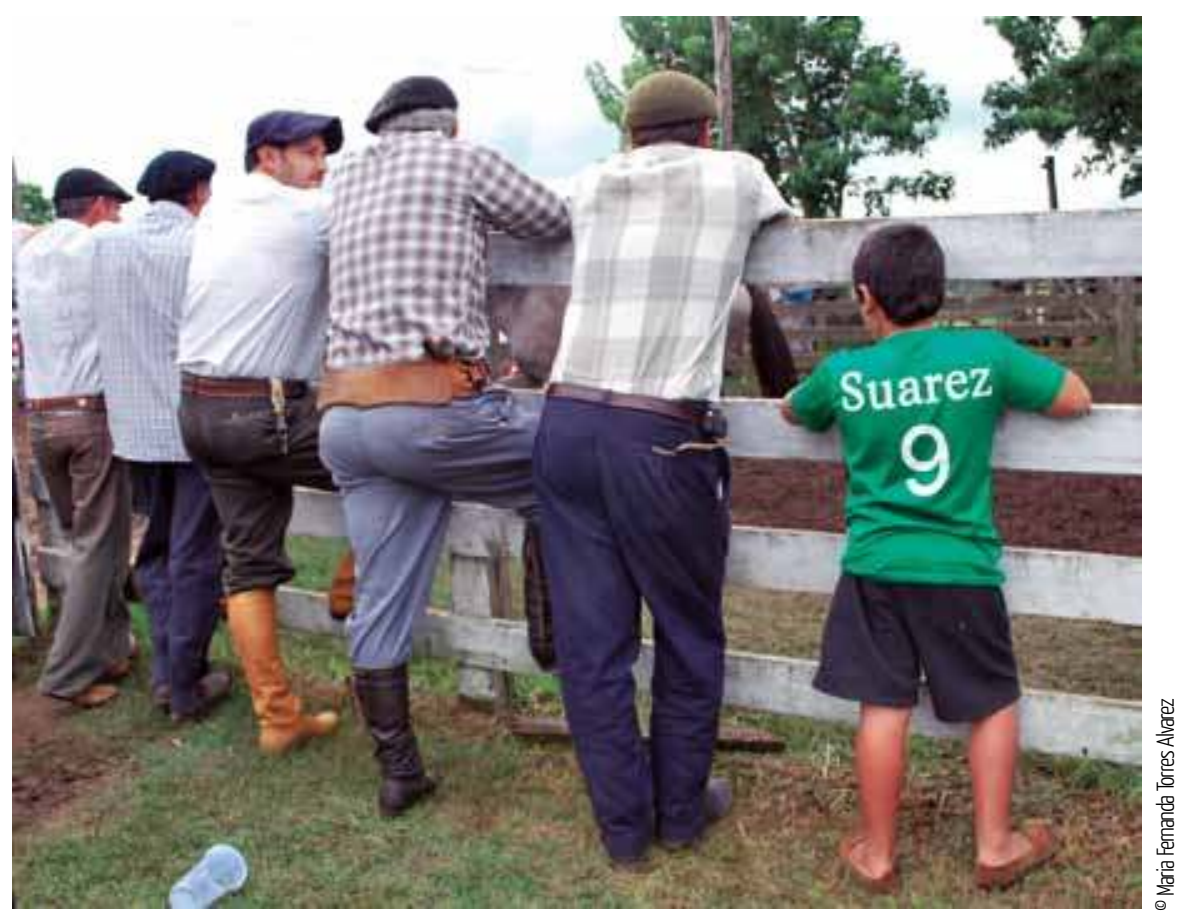

Au marché rural

Mai 2009, Département de Salto, Route 31, tentative équitable a remporté Carumbé, Uruguay.

Les peones ou les capataces, et plus encore les camperos sidentifient au gaucho. lls se sentent héritiers de sa vertu, de ses connaissances et de sa masculinité. Leurs couteaux, chapeaux, bottes, pantalons (bombachas), ponchos et chemises y font explicitement référence. 


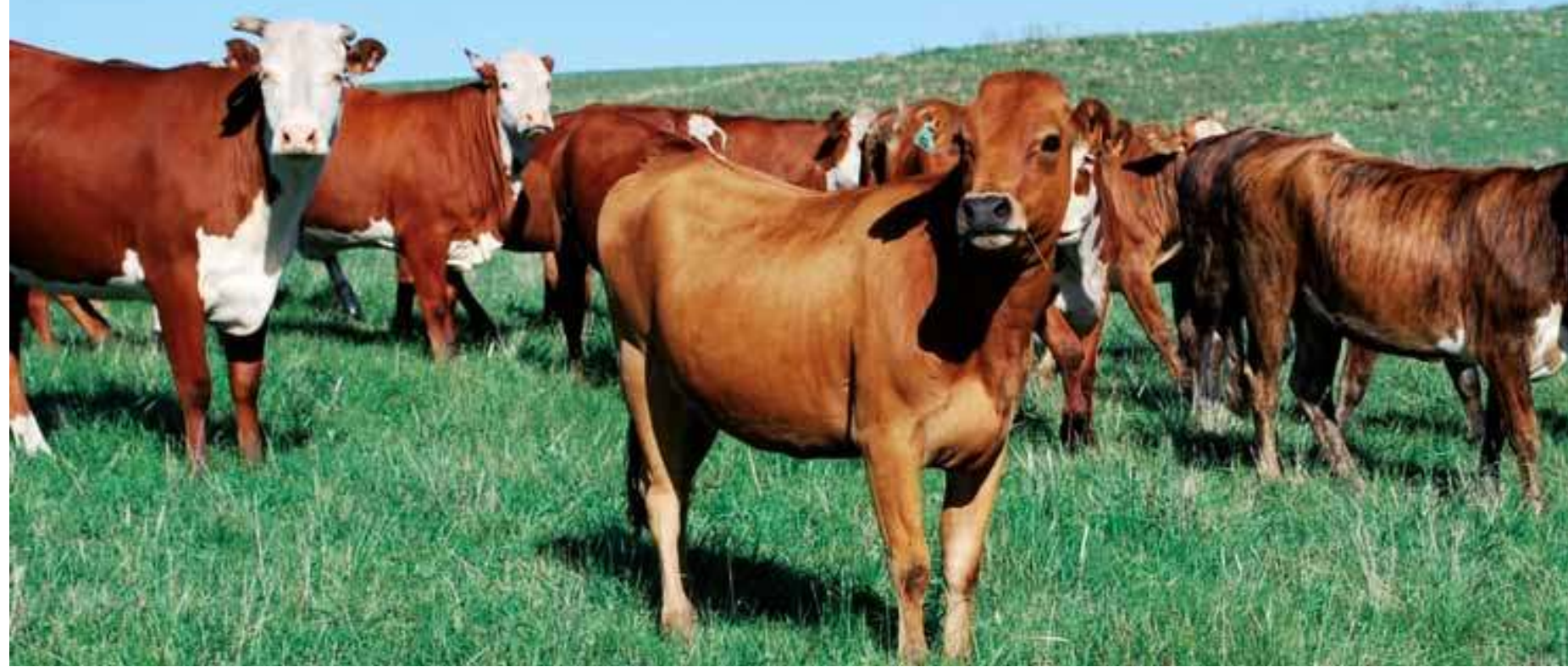

- Maria Fernanda Torres Alvarez

« au sud tout a subi un roulage, mais tu peux voir qu'ici c'est le contraire, ici il n'y en a pas, uniquement des petites parcelles qui sont complètement cultivées, c'est vrai... au sud tu ne trouves plus un terrain qui n'ait pas subi un roulage, au contraire de ce qu'on a ici $»^{3}$

Face aux recommandations des agronomes prônant une agriculture fourragère, beaucoup d'éleveurs font valoir une distinction nette entre les métiers d'éleveurs et d'agriculteurs et les compétences et connaissances respectives qui leur sont associés. La prise en charge des cultures ne fait pas partie de leur répertoire de connaissances, voire ne suscite pas d'intérêt de la part des éleveurs.

« j’ai un fils qui fait agronomie, mais il faut être ici, il faut se débrouiller pour être ici tous les jours, sinon voilà, ce qu'ils peuvent te dire, ça ne sert à rien ${ }^{4}$

« le conseil technique est tout ce qu'on a appris à faire ${ }^{5}$

Le conseil des agronomes vise à planifier, à rendre plus prévisible et à mieux contrôler, un monde affecté par des changements constants et complexes, alors que les recorridas visent précisément à mettre en relation tous les changements, à leur donner du sens, à les interpréter: enregistrer température, humidité, vent, hauteur et apparence des pâturages, condition des troupeaux. La recorrida, on y reviendra plus loin, s'oppose ainsi en tout point au travail de l'agronome qui consiste à maîtriser le sol et qui repose sur une approche de la terre comme une surface vide ou homogène. Cela suggère un travail qui serait panifiable et plus ou moins prévisible. Les planifications techniques prennent en compte les recommandations qui préconisent un recours aux produits chimiques engrais et glyphosate - et les espèces exotiques. Dans certains cas, ils sont attachés à une entreprise qui vend ces intrants. Par conséquent, leurs connaissances sont influencées par un intérêt commercial qui redouté par l'éleveur.

« il est difficile aujourd'hui de trouver un agronome qui n'est pas lié à une société de vente d'engrais, généralement tous les agronomes, au moins les plus accessibles ou ceux qui ont le plus d'expérience, travaillent tous avec une entreprise qui vend des engrais, qui vend des graines, ils ont tous des machines à louer ${ }^{6}$

\section{Braford}

Route 26, Paysandú, près de la ville Piedra Sola.

Ce sont des bovins Braford, un croisement entre la race britannique Hereford et indica de race Brahman. Ces choix sont récents dans le nord du pays, et ils sont choisis pour leurs caractéristiques rustiques qui leur permettent de mieux s'adapter à la sécheresse. Cependant, la plupart des rodéos dans le pays se déroulent avec la race Hereford, provenant de la politique publique pour moderniser les élevages de bovins au cours du xxe siècle. 


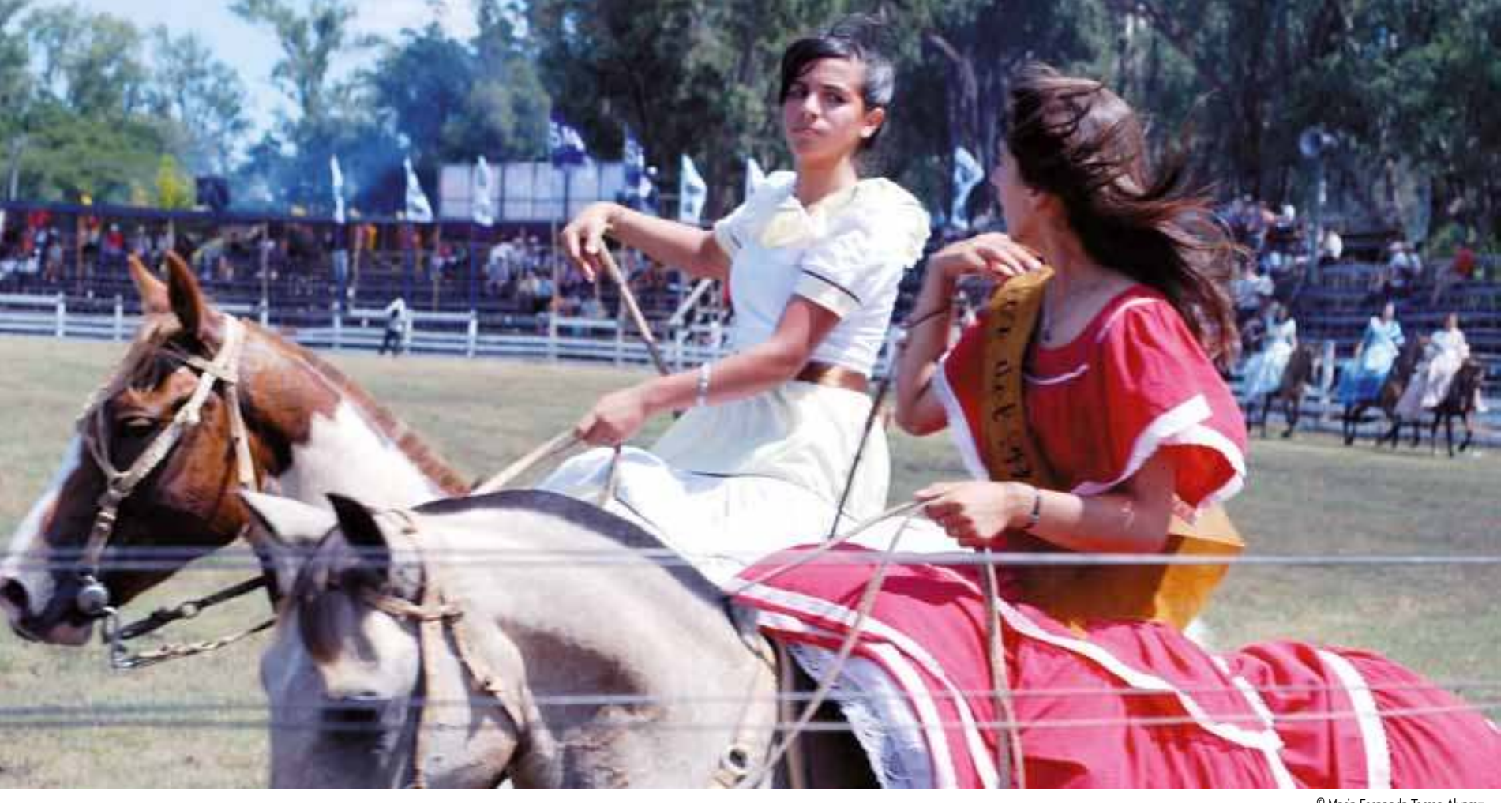

\section{Femmes en compétition pour la Fleur del Pago}

Mars 2011 dans la Fiesta Patria Gaucha, Département Tacuarembó.

C'est la plus grande célébration traditionnelle chez les professionels de l'élevage. Un moment est réservé au rôle des femmes, c'est le concours pour la Fleur del Pago, elles doivent exécuter plusieurs tests pour évaluer leurs compétences équestres et leurs connaissances des traditions. En règle générale, les femmes sont absentes des « paysages de l'élevage ».
« j'ai un ami qui est agronome, mais dès qu'il commence à parler avec moi il n'est plus un agronome, il est vendeur, parce qu'il a une entreprise qui vend des graines, des engrais... $»^{7}$

Cet intérêt commercial motive un rejet des conseils prodigués par les agronomes auxquels il est également reproché de ne pas connaître et de ne pas valoriser les espèces qui peuplent la prairie ${ }^{8}$. Le recours au semis-direct, c'est-à-dire un semis sans aucun travail du sol préalable, est une alternative qui n'induit pas de rupture du tapis végétal. La technique présente en revanche des contreparties car elle suppose l'utilisation de glyphosate, un herbicide non sélectif et favorise l'émergence d'organismes nuisibles qui s'installent après la destruction du tapis végétal.

Pour conclure, il apparaît clairement que la vision déployée par les agronomes s'appuie sur l'idée d'une amélioration synonyme d'interventions directes sur le milieu qui s'en trouve fortement modifié. Entièrement sous-tendue par la nécessité d'augmenter les rendements, un tel schéma relève nettement d'une volonté de maîtriser l'élevage conçu dès lors comme une «production » animale. C'est en cela que cette vision s'apparente au paradigme industriel du « making » formulée par Ingold « transformation, homogénéisation, rendement, maîtrise de tous les éléments du système (c'est-à-dire du milieu)» qui en sont les principaux mots éléments. Une telle vision suscite des critiques vives de la part des éleveurs qui défendent aujourd'hui un point de vue alternatif dont on peut trouver une première version au milieu du $x x^{e}$ siècle avec les Contributions de l'ingénieur Bernardo Rosengurtt qui a fortement contribué à décrire et légitimer l'intérêt de l'élevage traditionnel. 


\section{Les « Contributions »: légitimer et nommer « el campo »}

Un débat autour de ce qu'on a appelé le problème fourragé a timidement vu le jour pendant la première moitié du xxe siècle (1938-1948), à partir d'un ensemble de recherches sur le pâturage naturel, diffusées comme autant de Contributions ${ }^{9}$ rédigées par l'ingénieur agronome Bernardo Rosengurtt dans le but de mieux comprendre les attributs et la dynamique de pâturages naturels. Ses travaux ont été les premiers à mettre en relation la connaissance des travailleurs de l'élevage (peón, capataz et patron) et la connaissance scientifique. Il a ainsi montré que l'hétérogénéité des espèces est très élevée, selon le type de sol et les conditions climatiques, que la valeur nutritive du campo natural est meilleure que celles des cultures fourragères, et que le roulage détruit le tapis végétal et cause une érosion des sols (Gallinal $\&$ al., 1934). Les Contributions réfutaient donc point par point la thèse du problème fourrager national: elles préconisaient de conserver la qualité des pâturages naturels, dénommée par l'auteur capacité optimale, par rapport à la capacité maximale de production de l'animal (Rosengurtt, 1946). Elles ont de fait élargi le répertoire de description des pâturages naturels alors dénommés campos, par la caractérisation d'une grande diversité d'espèces (plus de 2000) qui se distinguent selon différentes associations végétales et sur différents types de sol et de climat (Rosengurtt, 1934, 1938, 1944). Avant les Contributions, l'écosystème était nommé en fonction des différentes espèces selon qu'elles étaient endémiques ou de substitution, c'est-à-dire importées (Trifolium repens L., Festuca arundinacea, Schreb, etc.). On parlait par exemple de prairie, pâturage, grass, pelouse, etc., mais jamais de « campo ».

Ce terme vernaculaire de campo est utilisé par les éleveurs et le camperos. Les Contributions le reprendront en lui conférant une identité singulière et une valeur intrinsèque qui s'opposera alors bien distinctement à l'idée d'homogénéisation propre à la logique industrielle. La controverse sur la dénomination de ces pâturages est tout sauf innocente. Elle a fait l'objet d'un récent accord de terminologie en écologie qui définit los campos comme suit: (n.) « pâturage naturel composé principalement de graminées, avec des herbes, arbustes et arbres occasionnels, dans des paysages vallonnés et montagneux, et une fertilité du sol variable ». Les campos forment la partie septentrionale de la Pampa dont le climat subtropical est humide, chaud en été et doux en hiver (Allen $\&$ al., 2011). Le terme diffère de fait de Cerrado, qui renvoie aux pâturages naturels des territoires situés plus au sud. Ils se caractérisent par un hiver plus long et plus sévère, ainsi qu'une abondance relative de légumineuses locales. Les campos forment la partie septentrionale de la Pampa. Le climat sub-tropical est humide, chaud en été et doux en hiver (Allen \& al., 2011)

Un autre apport majeur de ce travail a été de reconnaître la nature complexe du bétail (la modalité de sélection des pâturages à brouter est capricieuse, diffère selon chaque population, etc.). Dans les pâturages naturels, les effets du climat et de chaque type de sol (Rosengurtt, 1939 et 1943) ainsi que les différents systèmes de travail de chaque exploitation agricole. La complexité du système tient à la grande variabilité de chacune de ses composantes et la dynamique qui s'établit entre elles. Les Contributions recommandent ainsi de mettre à jour l'information des conditions quotidiennes de toutes les variables qui déterminent le pâturage naturel (Rosengurtt, 1946 et 1949). La seule façon de comprendre la dynamique globale de l'exploitation agricole est de se confronter dans la longue durée à cette variabilité, année après année, saison après saison qui voit alterner hivers froids et étés humides (Rosengurtt, 1946). C'est exactement ce qui caractérise le travail de la recorrida et les compétences de ceux qui s'y engagent, que Rosengurtt appelle, en lien direct avec le 
milieu naturel, les camperos. C'est à l'analyse de ce travail très particulier et des relations qu'il suppose avec le milieu et les bêtes que nous abordons ensuite.

Les Contributions proposent également une technique de gestion, c'est-à-dire, des améliorations de base, y compris les rotations, la plantation d'arbres pour s'abriter et produire de l'ombre pour l'élevage, et la subdivision des pâturages. Le principe d'innovation technologique, la capacité optimale comprend un double objectif: conserver les prairies naturelles et engraisser les animaux. Ce principe s'oppose à la capacité maximale qui a été promue par l'agronomie moderne et son « problème fourragé ».

\section{Habiter le campo natural, prendre soin des bêtes et de la prairie}

Derrière le terme recorrida se développe un savoir-faire, une compétence dans la gestion quotidienne de tous ses éléments (climat, le sol, l'herbe, les animaux) qui nécessite un investissement de temps en travail collectif impliquant des personnes expertes et apprenties, âgées et plus jeunes (de Torres, 2013).

« il y a le vieux campero, celui qui a vécu toute sa vie dans le pâturage naturel, le terme campero, je le dis toujours, ce qu'on trouve dans le dictionnaire est faux, parce qu'il dit tout sauf ce qu'il est, le campero n'est rien d'autre qu'une personne avec beaucoup d'expérience du pâturage et avec de l'intelligence, qui apprend toujours et qui capte des choses... c'est ça le campero, il sait comment dresser un cheval, monter à cheval... [des camperos] il y en a de moins en moins... ${ }^{10}$

Le campero est une personne (peón, capataz ou éleveur) expert sur la dynamique complexe de l'élevage en plein air dans une région particulière de l'Uruguay. En fait, cette figure professionnelle nécessite un long apprentissage auprès d'autres camperos âgés, lieux et moments d'une formation lente et progressive. Car tout est affaire de temps et d'expérience:

« se rendre compte de ce qui ne va pas, de ce qui est bien et quelles sont les choses qu'il faut corriger, c'est ce qui manque, il ne manque pas de connaissances, mais d'expérience pratique, on l'apprend avec quelqu'un qui a un savoir-faire, et qu'on acquiert après beaucoup de temps, c'est une affaire quasiment osmotique... on commence à l'apprendre... ce n'est pas comme si la personne qui a un savoir-faire peut s'asseoir et raconter, pas l'écrire, ni même la publier, c'est quelque chose à ressentir, à toucher » ${ }^{11}$

La recorrida ce n'est pas seulement un travail avec le bétail, mais avec le cheval, partenaire indispensable pour parcourir ces vastes espaces. La maîtrise de l'équitation s'impose, et dans tous ses aspects: dressage des chevaux et monte, qui s'apprennent dès la petite enfance. Les compétences du campero renvoient à une longue expérience de travail avec les animaux, à la nécessité de connaître l'histoire de chaque enclos, à la dynamique du pâturage et au climat local. Autant de prises sur le milieu naturel et de données, qui agencées 
les unes aux autres, vont permettre de comprendre les situations chaque fois nouvelles, et de vivre l'élevage jusque dans son corps. Car c'est le corps tout entier qui travaille. En effet, l'expertise mobilise tous les sens: la vue, l'ouie, l'odorat, le toucher qui sont autant de prises sur le milieu, permettant sa compréhension, son interprétation. Ainsi, l'humidité de l'air, la chaleur, le vent qui apporte les arômes et le son des textures contre lesquelles il se frotte, sont autant d'indices que le péon doit interpréter. Cette osmose avec le milieu n'a toutefois rien d'idyllique: les intempéries éprouvent les camperos, jusqu'à marquer leurs corps, burinés par le soleil et le vent, le travail forcé, par le froid et de chaleur.

Enfin, la recorrida met en jeu les compétences nécessaires pour comprendre le langage des animaux: être capable de communiquer avec le cheval, le chien et l'élevage. De telles connaissances s'incorporent dès l'enfance si l'on baigne dans «l'atmosphère » de l'élevage. Le terme renvoie ici fortement au weather-world développé par Ingold qui y voit une façon singulière d'habiter le monde et d'y développer des relations avec les autres, avec le milieu naturel dont l'élevage «à ciel ouvert » est un bon exemple. Un élevage infiniment réceptif et à l'écoute du milieu et de ses habitants: les vaches, chevaux et hommes confondus (Ingold, 2010).

Le travail de l'élevage consiste donc avant tout à veiller aux conditions environnementales nécessaires pour assurer le bon développement des animaux. Il s'agit d'accompagner la croissance de l'animal, de l'élever: de prendre soin aussi bien du bétail que du milieu naturel. Il relève en tout point de ce qu'Ingold désigne sous le terme de « growing », mis en opposition avec le « making » qui relève d'un registre plus interventionniste tel qu'il caractérise dans la conception de la moderniste de l'élevage décrite plus haut.

On comprend maintenant encore mieux la critique déployée à l'encontre de toute forme d'intervention directe sur le milieu qui s'accompagne d'une modification de l'écosystème naturel, comme le roulage évoqué plus haut et l'introduction d'espèces exotiques qu'il suppose:

« je n’utiliserai pas de rouleau par respect pour mes aînés et pour moi-même, j’ai grandi dans une famille de personnes qui conserve, qui a essayé de préserver les ressources naturelles, de les protéger et de les améliorer si possible, une famille qui pense qu'il ne faut jamais détruire une ressource naturelle pour obtenir un bien matériel, on a aujourd'hui le problème de l'exploitation minière, qui existe dans les pâturages, une grande partie du pays est perdue, il y a une loi qui permet aux entreprises minières de faire des fouilles pour essayer de trouver des minéraux ${ }^{12}$

Les agronomes, en particulier ceux qui vendent les intrants et les conseils techniques, n'habitent pas le campo: dépourvu de cette expérience intime qui fait du campero un expert à part entière, leur légitimité professionnelle est facilement remise en cause:

« Ils [les ingénieurs agronomes] ne sont pas au courant, ils ne savent pas ce qui se passe, ils examinent et ils partent, ils restent quelques mois et après, ce que la terre produit avec le climat est différent... ils ne savent pas... ${ }^{13}$

Autrement dit, le péon habite la pampa, autant que ses vaches et ses chevaux. Et c'est bien ce travail dans le temps long qui lui permet d'exercer son métier, et à l'élevage d'habiter la pampa.

Pour des raisons économiques pendant la révolution de l'indépendance, puis au nom du développement et de la modernisation au $\mathrm{xx}^{\mathrm{e}}$ siècle, les paysages agricoles de l'Uruguay ont tous été structurés par la volonté de substituer un écosystème primitif et donc 
improductif par une terre productive capable de suivre le rythme de la demande de l'industrie de la viande, notamment en lien avec les marchés internationaux. Lélevage moderne, industriel, intensif dans ses facteurs de production, s'est alors développé, fortement inspiré des pays du Nord: il a recours aux intrants, à des semences d'espèces exotiques, interventions directes sur le milieu. La logique industrielle à l'œuvre renvoie au paradigme du « making » (Ingold, 2010): il s'agit de fabriquer des animaux, qui sont avant tout envisagés comme de la viande et non pas forcément des êtres vivants. De la même façon les pâturages « artificiels » sont réduits à des facteurs de production et dépouillés eux aussi de toute dimension vivante. Dans une telle conception, la terre est considérée comme une surface, dont la capacité productive dépend d'autres facteurs de productions: intrants, techniques, semences et où la seule connaissance légitime renvoie à des savoirs directement liés aux marchés et à la volonté d'augmenter la productivité.

La modernisation de l'agriculture de l'Uruguay s'est fondée sur et s'est accompagnée de «l'invisibilisation » conjointe de l'écosystème naturel et du travail (sa valeur, ses compétences, sa complexité) des peones, des capataces et des éleveurs. On a montré que l'élevage extensif est caractérisé par ce que Ingold appelle " growing », autrement dit une capacité à élever des animaux, au sens fort du terme "élever », c'est-à-dire de les faire croître et de développer en tant qu'êtres vivants, dans un environnement à « ciel ouvert », avec tout ce que cela suppose de variabilité et de complexité. Loin de la vision disqualifiante des sociologues ruraux uruguayens, il apparaît que le travail de la recorrida est un travail à part entière fondé sur le soin (care), supposant une relation singulière et une familiarité avec les animaux (Porcher, 2011; Servière \& Dedieu, 2012).

Le rapport de force entre élevage traditionnel et élevage industriel, est, on le devine inégal. L'alimentation du bétail est la pierre d'achoppement de ces deux systèmes. L'élevage modernisé est basé sur la culture du soja qui permet de raccourcir la phase d'engraissement et d'augmenter les profits. Sa forme la plus industrielle est le «feedlot » que l'on trouve dans certains pays du Nord: les vaches sont maintenues dans des parcs d'engraissement et nourries au soja. La croissance économique assumée liée à l'expansion du soja et au développement des plantations forestières qui grignotent la pampa et empiètent sur le territoire de l'élevage a renforcé un imaginaire valorisant très fortement le progrès technologique permettant de hauts revenus, avec la promesse d'une ascension sociale.

\section{$\&$}

Nous avons montré comment deux points de vue sur les prairies et sur l'élevage uruguayens se sont constitués en opposition: celui des éleveurs et celui des agronomes. Chacun de ces points de vue défendant une conception particulière de ce que l'élevage 


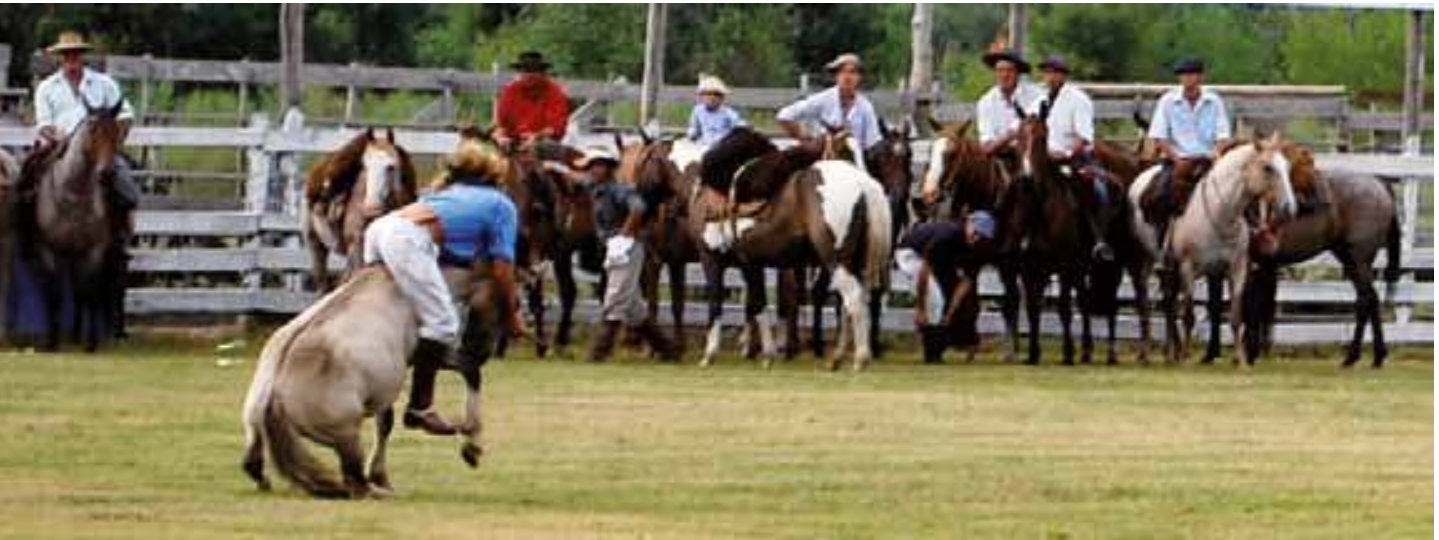

doit être: d'un côté un rapport au vivant animal et végétal basé sur un faible degré d'interventionnisme et la très forte valorisation des ressources locales de l'autre une approche fondée sur la nécessité de transformer le milieu et de contrôler la production, faisant appel à des technologies largement importées. Ces deux conceptions de la prairie naturelle reposent sur des connaissances qui ne bénéficient bien sûr pas de la même légitimité dans la construction de politiques publiques, dont dépendent le maintien et le devenir de l'élevage. L'implantation de l'industrie papetière, qui concurrence foncièrement l'élevage sur prairies naturelles, élargit et désectorialise la question de la productivité, pour la poser à l'échelle du territoire - un hectare d'eucalyptus étant plus rentable qu'un hectare de prairie naturelle.

À défaut du soutien des politiques sectorielles, c'est par la consommation, le changement climatique et la lutte contre la pauvreté que l'élevage traditionnel trouve un certain soutien. Tout d'abord, la demande constante des consommateurs en « viande naturelle » (carne natural) issue du « campo natural » est encouragée par l'élargissement des quotas de cette viande, ce qui a contribué à revaloriser l'élevage extensif sur pâturages naturels qui pénètrent les marchés internationaux. Ensuite, les politiques publiques dédiées à l'adaptation au changement climatique suggèrent la mise en œuvre de certaines des améliorations de base préconisées par le défenseur de la prairie naturelle Rosengurt ${ }^{14}$, qui ont été abandonnées en faveur de la proposition néo-zélandaise, dans la deuxième moitié du siècle dernier. S'il ne s'agit pas d'un retour en arrière, la reconnaissance de la légitimité de ces connaissances agronomiques - orientées aujourd'hui à la seule recherche d'une plus grande productivité - pourrait peut-être faire évoluer les débats académiques. Enfin, si elle n'y suffit pas, la lutte contre la pauvreté en milieu rural ne peut que concourir à soutenir l'élevage. Les deux derniers gouvernements de gauche ont cherché à améliorer la situation des travailleurs ruraux, notamment en instaurant la réglementation sur la durée quotidienne de travail (ramenée à huit heures), en encourageant l'action politique collective et en soutenant l'amélioration de la qualité de vie des populations rurales. À défaut d'être un soutien et une véritable reconnaissance professionnelle, cette politique de lutte contre la pauvreté pourrait, combinée aux deux autres dynamiques évoquées, contribuer à revaloriser les métiers de l'élevage. Comme dans beaucoup d'autres situations explorées dans ce Thema, les enjeux et les soutiens de l'élevage sur prairies
Mars 2011, la fête de la Patria Gaucha, Département Tacuarembó

La Fiesta de la Patria Gaucha met en vedette les compétences des hommes et des chevaux. La mise en place d'un rodéo, affiché comme un jeu - une exhibition, est là pour démontrer la force, le pouvoir et le degré de domestication des hommes sur les bêtes. 
naturelles gagnent à se désectorialiser mais la reconnaissance de cette activité ne peut faire l'économie d'un travail au sein du secteur lui-même permettant la reconnaissance de savoir-faire spécifique de l'élevage sur campo natural.

Ce travail a été conduit dans le cadre du projet de recherches MOUVE financé par l'Agence nationale de la recherche (projet ANR-2010-STRA-005-01).

\section{NOTES}

Photo d'ouverture: Des conditions climatiques extrêmes : Lair est frais, le soleil châtie les corps. Après la recorrida, la sueur de l'animal et celle de l'homme se fondent ; les arômes sont différents et forts. Les orages sont dantesques. Ils touchent le sol dans une furie qui illumine d'immenses régions à chaque éclair, plongeant tout le reste du territoire dans les ténèbres. La chaleur et le froid sont sans nuances. Le brûlant soleil estival change le vert en jaune et gêne le regard scrutant l'horizon. Le producteur malheureux attend, l'oreille collée à sa radio, l'annonce d'un épisode pluvieux. L'hiver, quant à lui, est dur. On y survit avec l'élevage. Photo de Maria Fernanda Torres Alvarez, pris en Septembre 2012 dans le département de champs Rivera (Route 29), l'Uruguay.

1. La Faculté d'Agronomie a été créée en 1908 sous l'impulsion des réformistes et progressistes de l'époque. Ils visaient le développement du potentiel de la terre afin d'augmenter les richesses du pays et de développer une civilisation européenne.

2. Homme de 45 ans, 22 ans d'expérience professionnelle, 500 hectares.

3. Patron de 68 ans, 36 ans d'expérience professionnelle, 1200 hectares.

4. Homme de 50 ans, 20 ans d'expérience professionnelle, 2200 hectares.

5. Homme de 45 ans, 27 ans d'expérience professionnelle, 1500 hectares.

6. Homme de 34 ans, 12 ans d'expérience professionnelle, 1800 hectares.

7. Homme de 50 ans, 30 ans d'expérience professionnelle, 2200 hectares.

8. Les cours sur la prairie à la Faculté d'Agriculture sont devenus facultatifs pour les étudiants et sont très peu fréquentés, d'après les enseignants. Certains agronomes connaissent toutefois bien les pâturages naturels et formulent des recommandations pertinentes pour une gestion appropriée des écosystèmes.

9. Les contributions sont une série de cinq livres et une conférence, réunissant les premiers travaux de recherche scientifique sur les attributs de la zone naturelle, financés par un des plus grands propriétaires fonciers dans le pays, avec le titre: "Études sur les prairies naturelles en Uruguay". Voir bibliographie.

10. Homme de 50 ans, 30 ans d'expérience professionnelle, 2200 hectares.

11. Homme de 34 ans, 12 ans d'expérience professionnelle, 1800 hectares.

12. Homme de 34 ans, 12 ans d'expérience professionnelle, 1800 hectares.

13. Homme 60 ans, 40 ans d'expérience professionnelle, 240 hectares.

14. Il s'agissait, grosso modo d'un système de rotations, d'arrosages et d'ombres (Rosengurt).

\section{POUR CITER CET ARTICLE}

Torres Alvarez de, M. -F. Prairie naturelle, Campero et ingénieur. In Dupré, L., Lasseur, J. \& R. Poccard-Chapuis Pâturages Techniques \& Culture 63: 74-91 


\section{RÉFERENCES}

Allen, VG. \& al. 2011 An Internatinal terminology for grazing lanas and grazing animals, Grass and Forage Science, 66, 2-28.

Böerger, A. 1939 Objetivos de la Comisión Nacional del Estudio del Problema Forrajero en el Uruguay. Buenos Aires: Facultad de Agronomía y Veterinaria-UBA.

de Torres Álvarez, M.- F. 2012 Tornar-se nativo/a resistência do liso. In Steil, C. \& de Moura Carvalho, I Cultura, percepção e ambiente. Diálogos con Tim Ingold. São Pablo: Terceiro Nome, CAPES.

- 2013 Guaridanes de la Pradera. El origen de la legitimidad de la ganadería en Uruguay. Tesis de Maestría en Sociología. Montevideo: Facultad de Ciencias Sociales, Universidad de la República.

de Torres Álvarez, MF, Toranza \& C. y Fourment, M. 2013 Campo Natural y Tecnologías natives para hacer ganadería. Primer Informe: genesis del campo natural. Montevideo: Fondo Bicentenario José Pedro Barrán-ANII.

Gallinal H., Bergalli S., Campal G. \& al. 1938 Estudios sobre Praderas Naturales del Uruguay. Primera Contribución. Montevideo: IGU.

Ingold, T. 2000 The Perception of environment: Enssays in Livelihood, Dwelling and Skill. Londres, Routledge.

- 2010 Footprints through the weather-world: walking, breathing, knowing. Journal of the Royal Anthropoloical Institute.

Ingold, T. \& Kurtilla T. 2011 Perceiving the Environment in Finnish Lapland, Body \& Society 6/November 2000 : 183-196< http://bod.sagepub.com/content/6/3-4/183> consulté le 3 février 2015.

Jobbágy, E. \& al. 2006 Forestación en pastizales: hacia una visión integral de sus oportunidades y costos ecológicos, Agrociencia 10: 109-124.

Moraes, M. - I. 2008 La Pradera perdida. Historia y economía del agro uruguayo : una visión de largo plazo 1760-1970. Montevideo: Linardi y Risso.

— 2001 Las determinantes tecnológicas e insitucionales del desempeño ganadero en el largo plazo, 18701970. Montevideo: Facultad de Ciencias Sociales -UdelaR (Tesis de Maestría Historia Económica).

Moraes, M.- I. \& de Torres, M.- F. 2013 Los Futuros del pasado. Informe interno el Núcleo de Estudios Sociales Agrarios. Montevideo: Facultad de Ciencias Sociales -UdelaR.

Moreira, B. 2004 Una nueva mirada de los empresarios ganaderos. Una descripción de los estilos de vida de los empresarios ganaderos de los departamentos de Durazno y Cerro Largo. Montevideo: Facultad de Ciencias Sociales -UdelaR (Tesis de grado en sociología).

Paruelo, J. \& al. 2006 Cambios en el uso de la tierra en Argentina y Uruguay: Marcos conceptuales para su análisis. Agrociencia X/2 : 47-61.

Piñeiro, D. 2008 El Trabajo precario en el campo uruguayo. Montevideo : Facultad de Ciencias Sociales -UdelaR.

Rearte, D. 2011 Informe de Evaluación Final del Programa de Apoyo a la productividad y desarrollo de nuevos productos ganaderos. Montevideo: Ministerio de Ganadería, Agricultura y Pesca.

Rosengurtt, B. 1939 La Variabilidad en la composición de las Praderas. Estudios sobre praderas naturales del Uruguay. Segunda Contribución. Rev. AIA, año XI, No.3: 28-33, Montevideo.

- 1943 La Estructura y el pastoreo de las praderas en la región de Palleros. Flora de Palleros. Estudios sobre praderas naturales del Uruguay. Tercera Contribución, Estancia Monzón-Heber, Estación Juan Jackson.

- 1944 Las Formaciones campestres y herbáceas del Uruguay. Estudios sobre praderas naturales del Uruguay. Cuarta Contribución. Estancia Monzón-Heber, Estación Juan Jackson.

- 1946 Estudios sobre praderas naturales del Uruguay. Quinta Contribución. Campo Experimental de Pastos. Estancia Rincón de Santa Elena, Estación Dr Alejandro Gallinal. Montevideo : Rosgal.

- 1949 Praderas Naturales: Los Problemas de su manejo. Apartado Revista de la Asociación de Ingenieros, Agrónomos 86/87. 


\section{RÉSUMÉ}

Prairie naturelle, « campero » et ingénieur. Deux conceptions de l'élevage en Uruguay. En Uruguay, la préservation de l'écosystème de prairie naturelle ne correspond pas à des politiques publiques environnementales d'avantgarde, mais à l'élevage sur pâturage naturel (campo natural). Les politiques publiques ont cherché à moderniser l'élevage, s'appuyant sur une légitimité agronomique qui considère les pâturages naturels comme étant de faible productivité. Ce projet de modernisation est opposé au maintien des pâturages naturels dans le territoire, lequel est lié à un élevage très saisonnier, où le travail principal (la recorrida) est assuré par des travailleurs agricoles dépourvus d'instruction scolaire Les différentes conceptions de la prairie naturelle reposent sur diverses formes de connaissances, qui structurent une hiérarchie des savoirs, lesquels bénéficient d'une légitimité plus ou moins grande pour orienter les politiques publiques. Deux points de vue sur les prairies se sont ainsi constitués: celui des éleveurs et celui des agronomes, chacun défendant une conception particulière de l'élevage: d'un côté un rapport au vivant animal et végétal basé sur un faible degré d'interventionnisme et la très forte valorisation des ressources locales, de l'autre une approche fondée sur la nécessité de transformer le milieu et de contrôler la production, faisant appel à des technologies étrangères.

\section{ABSTRACT}

Native grassland, the "campero" and the engineer. Two approaches to animal farming in Uruguay. In Uruguay, the preservation of the native grassland ecosystem does not correspond to avant-garde environmental public policies but, instead, to animal farming on native pasture (campo natural). Public policies have attempted to modernize animal farming by basing themselves on an agronomic legitimacy that links native grasslands to low productivity. This modernization project is at odds with the preservation of the country's natural grassland, which is linked to a highly seasonal type of animal farming in which uneducated farm workers are responsible for the majority of the work (la recorrida). The different approaches to native grassland are based on different forms of knowledge that structure a hierarchy of expertise and skills that benefit from a varying degree of legitimacy to shape public policies. Two viewpoints concerning natural grassland have therefore developed: that of livestock farmers and that of agronomists, each defending a particular approach to animal farming with, on the one hand, a relationship to living animals and plants based on a low degree of interventionism and a very high degree of development of local resources, and the other based on the necessity to transform the environment in order to control and increase production, making use of non-native technologies.

\section{MOTS-CLÉS}

élevage extensif, prairie naturelle, compétence pratique, connaissance agronomique

\section{KEYWORDS}

extensive breeding, native grassland, practical skills, agronomic knowledge 


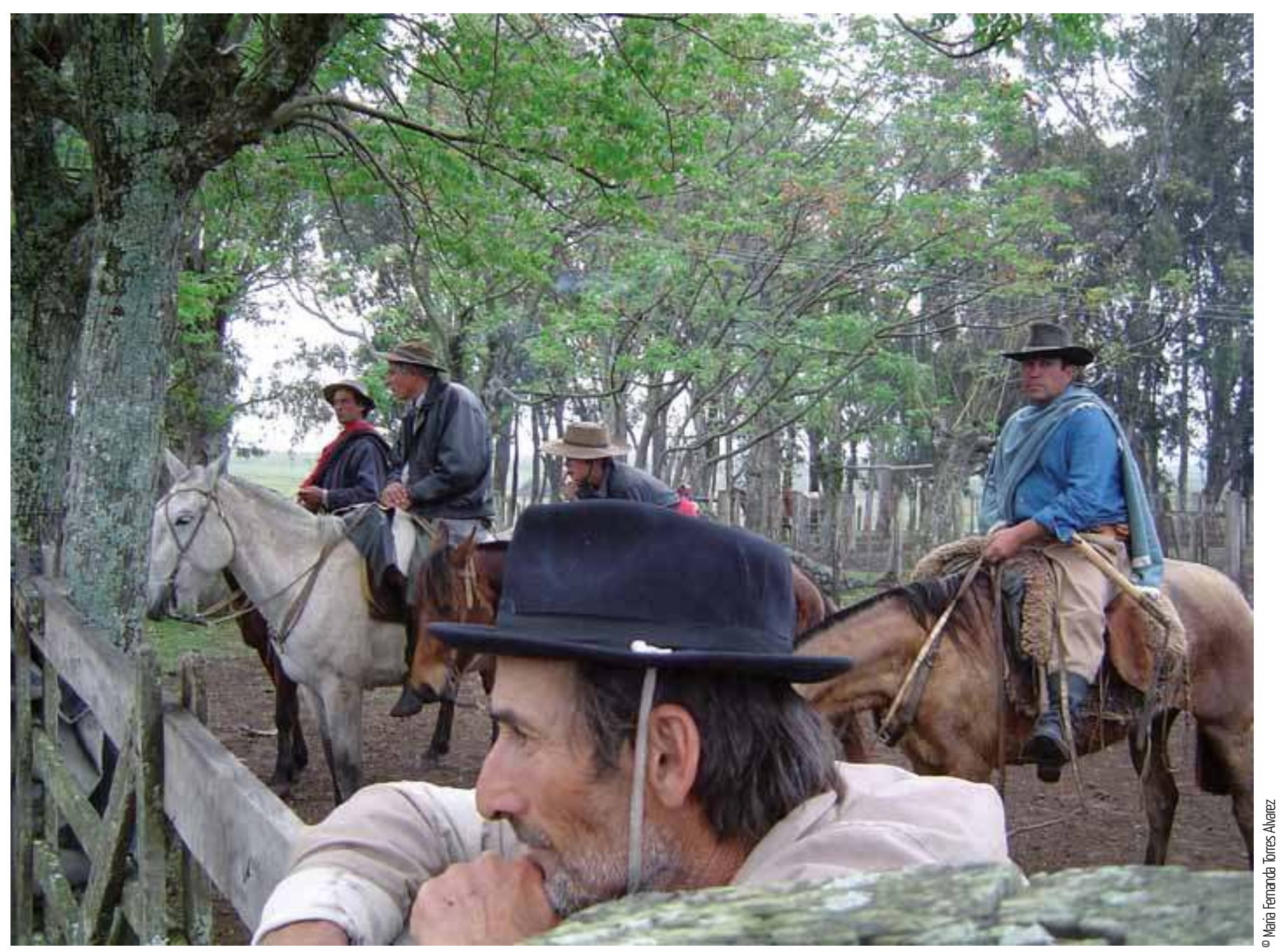

Bien quills partagent leurs

connaissances, les éleveurs

et les péones ne sont égaux

ni socialement, ni économiquement,

ni professionnellement.

L'asymétrie de leurs revenus en

témoigne. 